\title{
REM-CNC Router - CNC multifuncional Fresa e Laser de baixo custo construída reutilizando equipamentos de informática e lixo eletrônico
}

\section{Edinaldo Serra Cardoso Júnior ${ }^{1}$, Fernando Yoiti Obana ${ }^{2}$, Max Robert Marinho ${ }^{2}$, Lucas Kriesel Sperotto ${ }^{2}$, Sara Moreira da Silva ${ }^{3}$}

${ }^{1}$ Bolsista de Iniciação Científica 2019 - Cooperação FAPEMAT/UNEMAT - Campus de Alto Araguaia, MT, Brasil.

${ }^{2}$ Departamento de Computação, Universidade do Estado de Mato Grosso - UNEMAT, Alto Araguaia, MT, Brasil.

${ }^{3}$ Bolsista de Iniciação Científica 2019 - UNEMAT - Campus de Alto Araguaia, MT, Brasil.

edinaldo.junior@unemat.br, obana@unemat.br, max.marinho@unemat.br, sperotto@unemat.br, sara.moreira@unemat.br

\begin{abstract}
This paper describes the process of building a low cost multifunctional $C N C$ machine by reusing recycled materials. Called REM-CNC Router, this CNC machine has the ability to operate two non-simultaneous machine tools, allowing the engraving of images on the surface of materials by means of a laser, and the machining of printed circuit boards, 2D and even $3 D$ parts, depending on the complexity, using a cutter. Built with recycled material, it has a reduced cost becoming a CNC machine with a great cost benefit and production quality and besides cultivating the maker culture, it encourages the reuse of electronic waste and other recycled materials.
\end{abstract}

Resumo. Este trabalho descreve o processo de construção de uma máquina CNC multifuncional de baixo custo reutilizando materiais reciclados. Denominada de REM-CNC Router, essa máquina CNC possui a capacidade de operar duas máquinas-ferramentas não simultâneas, permitindo a gravação de imagens na superfície de materiais por meio de um laser, e a usinagem de placas de circuito impresso, peças em $2 D$ e até mesmo $3 D$, dependendo da complexidade, utilizando uma fresa. Construída com material reciclado, possui custo reduzido tornando-se uma máquina CNC com um ótimo custo benefício e qualidade de produção e além de cultivar a cultura maker, incentiva a reutilização de lixo eletrônico e outros materiais reciclados.

\section{Introdução}

Nas últimas décadas, tem ocorrido um avanço tecnológico crescente que teve como consequência a substituição de maquinas-ferramentas operadas manualmente por maquinas-ferramentas automatizadas. Esse processo acabou originando a tecnologia NC (Numeric Comands, ou Comandos Numéricos) por volta de 1949, que posteriormente, progrediu para CNC (Computer Numerical Comands, ou Comando Numérico Computadorizado). 
A primeira máquina $\mathrm{CNC}$ foi desenvolvida no Instituto de Tecnologia Massachusetts (MIT), e somente por volta de 1982, uma década depois de expandir-se nos setores da indústria, popularizou-se entre as empresas de médio porte por conta da introdução de computadores de custo reduzido (LEITE et al., 2017).

Posteriormente denominada de Computer Numerical Control ou Controle Numérico Computadorizado, esta tecnologia pode ser definida como um método avançado de automação, que por meio de instruções, permite o controle da movimentação e operação de máquinas-ferramentas, tendo como principais vantagens a eficiência, flexibilidade, redução de custo e tempo, e precisão (VERMA; GIANCHANDANI; SAROHA, 2018).

Com o constante avanço da tecnologia e da economia, surgiram novos requisitos industriais, como aumento da precisão, qualidade, altas taxas de produção e baixos custos que conseguem ser atendidos somente com o uso da tecnologia $\mathrm{CNC}$, por não possuir as limitações humanas e ser capaz de fabricar produtos altamente precisos em escalas nanométricas de maneira muito mais ágil e rápida (JAYACHANDRAIAH et al., 2014).

Atualmente a tecnologia CNC é amplamente utilizada nas indústrias, principalmente na aeronáutica e no automobilismo, aumentando a precisão e qualidade dos produtos e permitindo a fabricação de peças extremamente complexas. Entretanto, essa tecnologia possui um custo muito elevado tornando-se inacessível a maior parte das pessoas. Porém, com o avanço da internet na última década e a popularização da cultura maker, diversos projetos em escala menor puderam ser reproduzidos baseados na tecnologia CNC usada nas grandes industrias, tornando-a acessível e permitindo que estudantes e simpatizantes da tecnologia desenvolvessem seus próprios projetos (DANTAS; JUNIOR, 2016).

Segundo Cardoso Júnior (2019), o problema mais comum no desenvolvimento de sistemas robóticos e de automação, é o alto custo na obtenção de materiais essenciais para a montagem desses sistemas. Uma solução para este problema é a reutilização de materiais eletroeletrônicos, esta prática reduz o custo de aquisição de alguns materiais para zero (CARDOSO JÚNIOR et al., 2019). Além da redução dos custos, a reutilização e reciclagem de eletroeletrônicos, diminui a produção de resíduo eletrônico e reduz impactos no meio ambiente (OBANA et al., 2018).

Com base nesses pontos e com a necessidade da produção de placas de circuito impresso de alta qualidade, usinagem e corte a laser de peças para uso em diversos projetos do LESE (Laboratório de Eletrônica e Sistemas Embarcados), surgiu a motivação para a construção de uma máquina $\mathrm{CNC}$ multifuncional de médio porte utilizando materiais reciclados, que recebeu o nome de REM-CNC Router (Recycled Material-CNC Router). Essa máquina $\mathrm{CNC}$ poderá ser usada tanto como uma router $\mathrm{CNC}$ quanto como uma Laser CNC, permitindo a fabricação de uma gama de produtos com qualidade profissional em menos tempo e com custo reduzido, além de diminuir os impactos ao meio ambiente pelo fato de ser construída reutilizando lixo eletrônico e outros materiais.

\section{Metodologia}

A principal referência para a montagem da REM-CNC Router, foi o projeto CNC 2.1 Caseira com Arduino com Trilhos de Gavetas Telescópicos de Baixo Custo de Marlon Nardi Walendorff sob a Licença Creative Commons - Atribuição-Não Comercial 4.0 Internacional, podendo ser acessado no endereço eletrônico https://www.marlonnardi.com/p/construa-sua-propria-cnc-20-2.html. 
Analisando o projeto de Walendorff (2020) e levando em consideração que a proposta do projeto já era de baixo custo, percebeu-se que haveria uma redução de custos se fossem utilizados materiais reciclados para a construção da máquina CNC. Verma (2018) afirma em seu estudo, que o maior benefício na utilização de material residual de dispositivos eletroeletrônicos na construção de uma máquina $\mathrm{CNC}$, é a relação custo-benefício e a diminuição de custo (VERMA; GIANCHANDANI; SAROHA, 2018).

\section{Fundamentação Teórica}

\subsection{Tipos de máquinas $\mathrm{CNC}$}

De acordo com Polastrini (2016), uma máquina CNC é qualquer equipamento eletrônico que guia ferramentas ao longo de trilhos dispostos em um plano de coordenadas cartesianas. Essas máquinas utilizam informações geométricas inseridas em um sistema de coordenadas cartesianas para se movimentar nos eixos, podendo ter como referencial para percorrer as dimensões da peça de forma precisa, a coordenada inicial da peça ou a coordenada inicial de trabalho da máquina (GOBI, 2019).

Esses movimentos consistem na interpretação do arquivo $G$-code pela máquina que pode variar de acordo com a necessidade de uso, como por exemplo, máquina de corte a laser, fresadora, router, gravadora de circuitos eletrônicos, dentre outras inúmeras aplicações (POLASTRINI, 2016). Tendo em vista os problemas apresentados no Tópico 2, o diferencial da REM-CNC Router é a utilização de dois tipos de máquinas CNC integradas em uma única máquina, sendo elas a router $\mathrm{CNC}$ e a laser $\mathrm{CNC}$, permitindo a troca das funções de uma forma simples e compacta.

\subsubsection{Router CNC}

Uma router $\mathrm{CNC}$ é projetada para usinar materiais de baixa densidade como madeiras, ligas de alumínio e polímeros, tendo como prioridade tanto a força de tração quanto a velocidade do deslocamento da ferramenta nos eixos do plano cartesiano, podendo variar de acordo com o material a ser trabalhado. Sua forma de trabalho consiste na fixação do material, a ser usinado, em uma mesa, que possui em suas laterais, trilhos fixos que garantem a mobilidade e estabilidade do conjunto de deslocamento do eixo X. Um segundo seguimento de trilhos fica posicionado perpendicular ao eixo $\mathrm{X}$ permitindo o deslocamento do eixo $\mathrm{Y}$, onde ficam posicionados trilhos na vertical, garantindo a movimentação do conjunto de ferramentas acopladas ao eixo Z (POLASTRINI, 2016).

\subsubsection{Laser CNC}

Uma Laser CNC possui praticamente as mesmas características de uma router $\mathrm{CNC}$, podendo ser diferenciado apenas por não possuir o eixo Z. No lugar de um motor ou micro retifica, é utilizado um módulo de raio laser com capacidade para fazer cortes de materiais ou gravações na superfície de contato, a espessura de corte ou profundidade da gravação é determinado pela potência do laser refletido sobre o material trabalhado. Normalmente as máquinas laser $\mathrm{CNC}$ possuem apenas dois eixos de deslocamento, e tem como prioridade a velocidade de deslocamento da ferramenta nos eixos $\mathrm{x}$ e $\mathrm{y}$ (POLASTRINI, 2016). 


\subsection{Hardware}

O hardware é o responsável por fazer o controle do deslocamento dos eixos no plano cartesiano baseado em instruções recebidas por um software. Existem diversos tipos de hardware capazes de executar essas funções dependendo da necessidade de sua aplicação. Como exemplo, podemos citar os CLP's (Controladores Lógicos Programáveis) que são um sistema de microprocessadores que executam uma função de controle logico, aritmético, de temporização e sequenciamento, baseado em informações de entrada que permite a manipulação de relés de saída. Amplamente utilizados nas industrias, os CLP's possuem uma grande variedade de hardware que podem ser utilizados em diversas situações, porém por possuir um custo muito elevado, seu uso torna-se inviável em projetos de pequeno e médio porte.

Além dos CLP's existem os Microcontroladores, que são um circuito integrado programável que possuem CPU (Central Process Unit ou Unidade Central de Processamento), memória, portas de entrada e saída, dentre outros componentes, possibilitando sua aplicação desde um simples controle remoto a maquinas extremamente complexas.

Um dos microcontroladores mais utilizados na comunidade DIY (do it yourself) é o AVR ${ }^{\circledR}$ da $A t m e l \circledR$, que faz parte da composição da placa de prototipagem eletrônica Arduino, sendo muito popular pelo seu baixo custo e a facilidade de aplicação em projetos pequenos e até mesmo mais complexos (PALMIERE, 2016).

As vantagens citadas por Palmiere (2016) tornaram o Arduino a opção mais viável para ser utilizado na construção da REM-CNC Router, por possuir um custo relativamente baixo, Hardware e Software livre e uma variedade de placas com modelos diferentes que podem ser utilizadas de acordo com a necessidade de cada projeto (CARDOSO JÚNIOR; OBANA, 2018).

\subsubsection{Arduino UNO REV3}

A placa escolhida para o desenvolvimento do projeto foi a Arduino UNO REV3 com microcontrolador ATmega328P, que possui 14 pinos de entrada e saída digital dos quais 6 são PWN (Pulse Width Modulation), 6 entradas analógicas, conexão USB (Universal Serial Bus), um conector de alimentação que pode variar de $5 \mathrm{~V}$ a $12 \mathrm{~V}$, um ressoador de cerâmica de $16 \mathrm{MHz}$ (CSTCE16M0V53-R0), um botão de reinicialização e um conector ICSP (ARDUINO UNO REV3 | LOJA OFICIAL DO ARDUINO, 2020).

O Arduino recebe por meio da porta USB o $G$-code, que possui as instruções para realizar o processo de comando da máquina $\mathrm{CNC}$, interpreta o $G$-code e aciona os atuadores que comandam a movimentação da ferramenta nos eixos de movimentação (POLASTRINI, 2016). Além disso, o Arduino possui uma vasta extensão de códigos e bibliotecas prontas que são disponibilizadas por comunidades ligadas a plataforma, podendo ser alteradas por usuários com conhecimento de programação em linguagem de alto nível (GOBI, 2019).

\subsubsection{CNC Shield V3}

De acordo com Polastrini (2016) uma forma de melhorar o funcionamento, manutenção e evitar erros e falhas em máquinas CNC é a utilização de uma CNC Shield em conjunto com o Arduino UNO. Estes Shields podem ser facilmente interligadas ao Arduino e expandem a capacidade de conexões, facilitando o uso de displays e outros módulos 
compatíveis com a placa (POLASTRINI, 2016). O CNC Shield V3 é um módulo compatível com o Arduino UNO REV3 e possui conexões de entrada e saída para o controle de componentes como, Motor de passo, módulos Endstops, Spindle e outros. Amplamente utilizado para máquinas $\mathrm{CNC}$ e impressoras 3D, o CNC Shield é controlado por um firmware que fica armazenado na memória do Arduino que é responsável por converter o G-code em movimentos mecânicos dos motores de passo conectados ao CNC Shield (ANIS; SANTOSA, 2019).

Um dos benefícios de se utilizar o CNC Shield V3, é a simplicidade da instalação eletrônica, ele possui encaixes para a conexão direta de até quatro drivers de potência e uma entrada de alimentação independente para esses drivers, que pode variar de $12 \mathrm{~V}$ a 35V (POLASTRINI, 2016).

\subsubsection{Drivers de potência $A 4988$}

Para fazer o controle dos motores de passo de forma que sejam garantidos a precisão, o torque e a velocidade de operação adequada, é de grande importância um bom circuito de acionamento que possua essencialmente uma dupla ponte $\mathrm{H}$ para controlar os motores em acionamento bipolar. Cada ponte $\mathrm{H}$ é responsável por uma bobina do motor. Esses circuitos são desenvolvidos utilizando transistores do tipo MOSFET (Metal Oxide Semiconductor Field Effect Transistor), que são altamente indicados por possuírem frequência de acionamento acima de $500 \mathrm{kHz}$ e realizarem o controle, por tensão, da passagem de corrente pelo transistor. Este projeto utilizou o driver A4988, por ser de fácil operação, permitir o controle de motores de passo que possuem acionamento bipolar, e ser construído com MOSFET. É um dos drivers mais utilizados no desenvolvimento de máquinas CNC e impressoras 3D (MOREIRA, 2018).

O driver A4988 possui um sistema ajustável de corrente, permitindo o usuário controlar a saída máxima de corrente por meio de um potenciômetro presente no próprio driver. Com isso é possível ajustar a tensão adequada para o motor alcançar velocidades maiores sem o risco de dano, podendo operar em até cinco níveis de resolução sendo de passo completo, $1 / 2$ passo, $1 / 4$ de passo, $1 / 8$ de passo e $1 / 16$ de passo, além de operar em tensões que variam de $8 \mathrm{~V}$ a $35 \mathrm{~V}$ e chavear $1 \mathrm{~A}$ por fase (MOREIRA, 2018).

\subsection{Motor de Passo}

Atuadores são dispositivos eletromecânicos que transformam cadeias de pulsos elétricos em energia mecânica, permitindo o movimento rotacional discreto de um eixo podendo ser pequenos incrementos angulares, denominados de passo (MOREIRA, 2018). O motor de passo é formado por um conjunto do qual fazem parte o rotor e o estator, que é composto por pares de bobinas de cobre conectadas e posicionadas em sentido contrário umas das outras, possuindo suas extremidades ligadas aos terminais do motor. Denominadas de fase, cada conjunto de bobinas quando energizadas provocam interações eletromagnéticas no interior do motor, fazendo com que o rotor gire. Para que o giro do rotor possa ser controlado, o motor é alimentado com sequências de pulsos elétricos ordenados em cada uma de suas fazes, gerando o movimento angular do rotor que é denominado de passo e determinando o sentido de giro do rotor (POLASTRINI, 2016). Motores de passo possuem alto torque, sincronismo, ângulo de rotação e posicionamento preciso (MOREIRA, 2018). 


\subsection{Softwares}

O software é responsável pela interface entre o usuário e a máquina, permitindo o controle e manipulação das ações da mesma por meio de uma interface gráfica intuitiva e de fácil operação (FACHIM, 2013). Além disso, é necessário que esse software envie os comandos que serão interpretados pelo firmware tornando possível o controle dos atuadores. Esses softwares são denominados de CAM (Computer-Aided Manufaturing) e são amplamente utilizados pelas maquinas CNC's, fornecendo as instruções que deverão ser seguidas pela máquina para iniciar e concluir o processo de fabricação de um determinado produto. Existem variados tipos de softwares CAM open source que possuem compatibilidade com Grbl (interpretador de G-code), que podem ser utilizados para fazer o controle e configuração do G-code (MOREIRA, 2018).

\subsubsection{Firmware Grbl}

O firmware Grbl é um interpretador de G-code criado por Simen Svale Skogsrud em 2009, e por ser de código aberto é muito utilizado pela comunidade DIY (FACHIM, 2013). Desenvolvido e otimizado para ser utilizado em conjunto com microcontroladores ATmega328p, é o responsável por controlar os movimentos da máquina. É um software livre desenvolvido em linguagem $\mathrm{C}$, possui licença GPLv3, requer hardware simples e tem padrões industriais, tornando-se base para máquinas $\mathrm{CNC}$, impressoras $3 \mathrm{D}$ e outras máquinas de três eixos (POLASTRINI, 2016).

O papel desempenhado pelo Grbl instalado em uma placa Arduino com microcontrolador ATmega328p, é receber, por meio da porta USB (Universal Serial Bus), um conjunto de instruções enviadas em $G$-code pelo computador, interpretá-las, e transmitir comandos para os drivers de potência e atuadores que transformarão os sinais elétricos em movimentos. O Grbl possui comandos de operação das maquina-ferramentas como velocidade, liga/desliga e algumas interações durante o processo como pausa e interrupção (MOREIRA, 2018).

\subsubsection{G-code}

As máquinas CNC seguem uma padronização composta por linhas de instruções denominadas de blocos, que são formadas de palavras que possuem letras e números. Esse padrão denominado de Código G, é definido pela normativa da ISO 6983.1 e é composto por duas categorias básicas de funções, as funções preparatórias $(\mathrm{G})$, cujo papel é indicar para a máquina-ferramenta como ela deve operar deixando-a pronta para executar movimentos, e as funções miscelâneas que são complementares às funções preparatórias e geralmente atuam como chaves programáveis de componentes da máquina, como fusos, motores e outros sistemas auxiliares (REZENDE et al., 2019).

\subsubsection{IDE do Arduino}

O Arduino pode ser controlado através de um conjunto de instruções enviados para o microcontrolador da placa, por meio da linguagem de programação Arduino baseada em Wiring e utilizando o Software Arduino (IDE - Integrated Development Environment ou Ambiente de Desenvolvimento Integrado) baseado em Processing. Além de possuir plataforma cruzada, o que permite a execução em diversos sistemas operacionais, a IDE do Arduino é fácil de ser operado permitindo o uso tanto de usuários iniciantes quanto de 
usuários avançados, podendo ser expandida por meio de bibliotecas $\mathrm{C}++$ (ARDUINO, 2020).

\subsubsection{Universal GcodeSender}

Para que o computador e a máquina se comuniquem, é fundamental o uso de um software que envie o conjunto de instruções que o firmware irá ler e executar. Compatíveis com o Grbl, existem diversos softwares que o usuário pode escolher para desempenhar esta função, tais como o bCNC, Easel e o Universal GcodeSender. Desenvolvido em Java sob a licença GPLv3, o Universal GcodeSender possui compatibilidade com vários sistemas operacionais como Windows ${ }^{\circledR}$, Linux e Raspberry, é de fácil configuração e o mais utilizado. O Universal GcodeSender apenas faz a leitura do $G$-code e o envia para a máquina CNC, não possuindo suporte para criação ou edição de G-code (POLASTRINI, 2016).

\subsubsection{LaserGRBL}

O LaserGRBL é capaz de gerar e transmitir os comandos e trajetória do G-code para o Arduino diretamente, o que permite gravar imagens, fotos e logotipos por meio de uma ferramenta de conversão interna que o software possui. Sendo diferente de outras GUI (Graphical User Interface ou Interface Gráfica do Utilizador ou Usuário), o LaserGRBL foi desenvolvido para uso com cortador e gravador hobbist a laser, e para que sejam utilizados todos seus recursos, o seu gravador deve fornecer suporte à modulação de potência por meio do comando S. Possui compatibilidade com o firmware Grbl v0.9 e Grbl v1.1 e é uma ferramenta de fácil uso e gratuita (LASERGRBL, 2020).

\section{Desenvolvimento}

Para a construção da máquina $\mathrm{CNC}$, foram utilizadas como base, instruções disponibilizadas por Walendorff (2020) com o título de "Construa sua própria CNC 2.1", que disponibiliza todas as etapas de montagem desde a estrutura física até a mecânica e eletrônica. Inicialmente foi necessário um levantamento de peças e componentes viáveis que poderiam ser utilizados para execução deste projeto, tendo em vista que o objetivo deste trabalho é reduzir os custos na construção de máquinas $\mathrm{CNC}$ reutilizando material reciclado. A aquisição dos componentes mecânicos e eletrônicos necessários se deu por meio do Projeto de Reutilização e Reciclagem de Equipamentos de Informática (OBANA et al., 2018), o que permitiu redução nos custos de construção da máquina CNC. Durante as análises do projeto de Walendorff (2020) e o projeto da REM-CNC Router foram realizadas a substituição de alguns materiais e a alteração de algumas estruturas físicas, para aumentar a estabilidade e a área de trabalho da máquina CNC (Figura 1). 

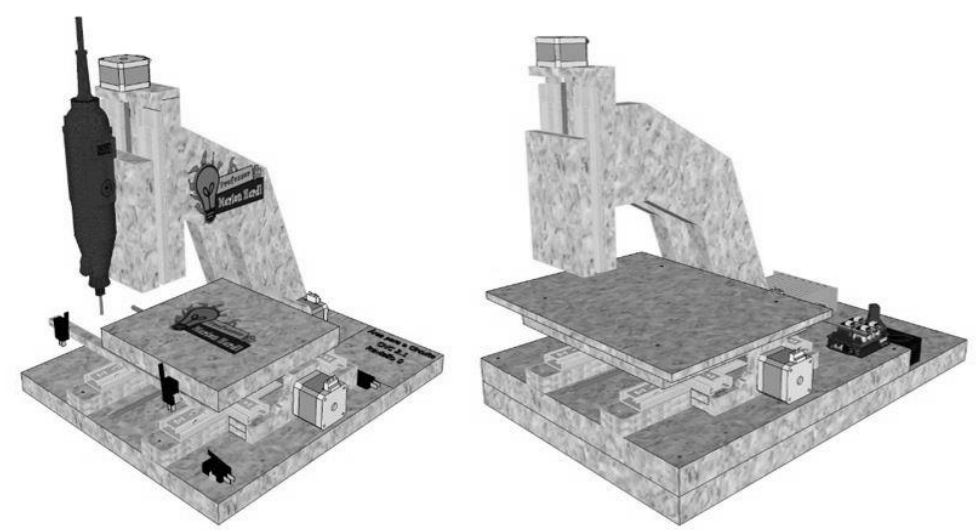

Figura 1. Modelo 3D do projeto original (esquerda), e Modelo 3D da REM-CNC Router (direita).

Fonte: Autor (2020)

Comparando as imagens da Figura 1, percebe-se que houve um aumento no porte da máquina, bem como um aumento da área de trabalho. Tomando como referência as medidas das peças disponíveis por Walendorff (2020), foi adicionada uma base com medidas de $35 \times 55 \mathrm{~cm}$, fabricadas em MDF com espessura de $30 \mathrm{~mm}$ para acoplar a Peça 1, também fabricada em MDF com espessura de $30 \mathrm{~mm}$, o que permitiu maior estabilidade durante a operação da máquina. Para que houvesse o aumento da área de trabalho da máquina $\mathrm{CNC}$, foram alteradas as medidas das peças 2,3 e 4 gerando a necessidade de serem adicionadas novas peças que podem ser analisadas nas (Figura 2).

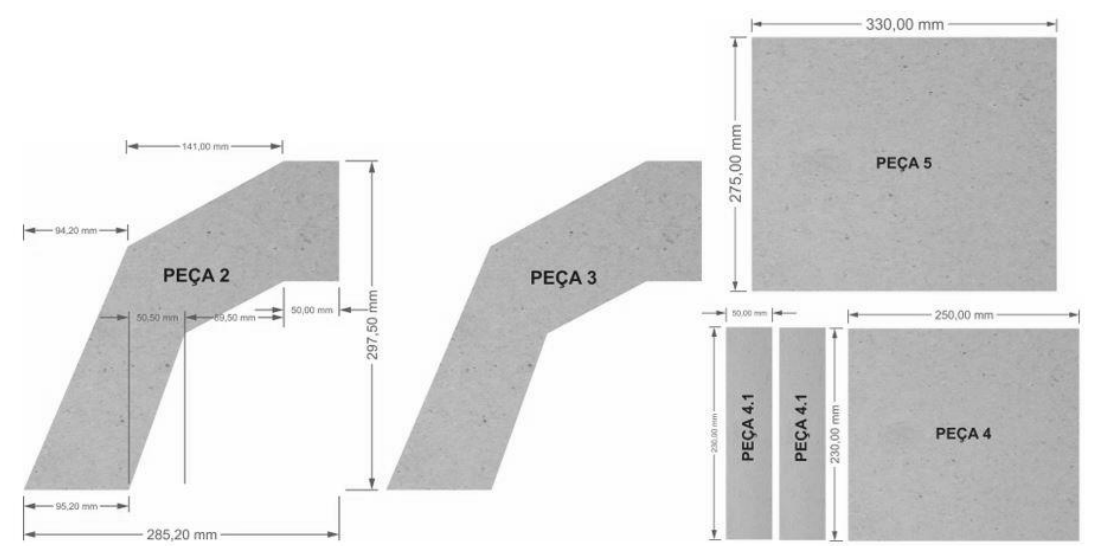

Figura 2. Peças 2, 3 e 4 com novas medidas e novas peças

Fonte: Autor (2020)

Essas alterações foram realizadas por conta da necessidade de uma máquina CNC com uma área de trabalho maior, sendo que o projeto original permitia uma área de trabalho de 200x200 mm e a REM-CNC Router possui uma área de trabalho de 250x250 mm. Além das alterações na parte da estrutura física, foram feitas alterações na parte mecânica visando maior durabilidade. Durante a preparação dos trilhos telescópicos baseado em Walendorff (2020), percebeu-se que haveria um descarte de material metálico proveniente do corte dos trilhos. Devido a este corte, pôde ser feita a substituição da placa de fenolite, utilizada para construir a base que serviria para a movimentação dos eixos $\mathrm{X}$ e Y, por essa sobra de material metálico proveniente do corte dos trilhos (Figura 3). 


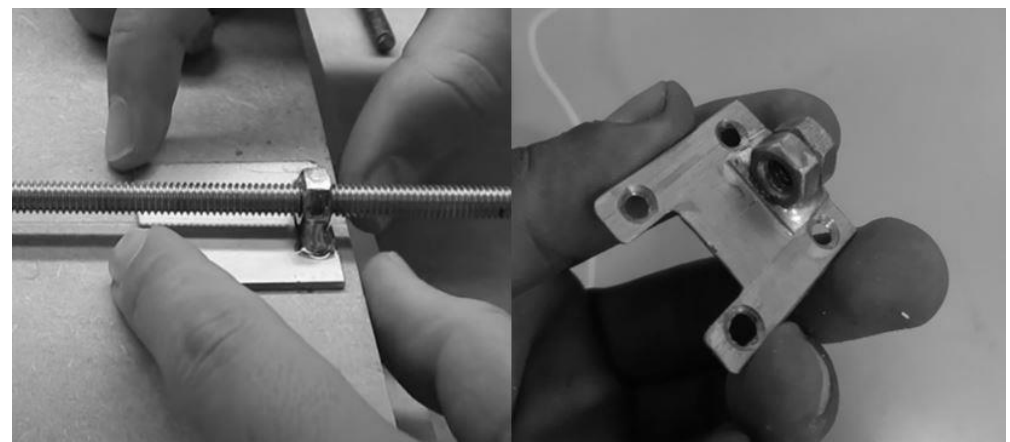

Figura 3. Peça do projeto original feita com placa de fenolite (esquerda), Peça adaptada feita com sobra de trilho telescópico (direita).

Fonte: Autor (2020)

Uma outra alteração feita no projeto original, foi a substituição do Espaguete Termo retrátil utilizado para a junção da barra roscada ao eixo do motor, por uma mangueira para gasolina em silicone, permitindo maior flexibilidade e durabilidade (Figura 4).

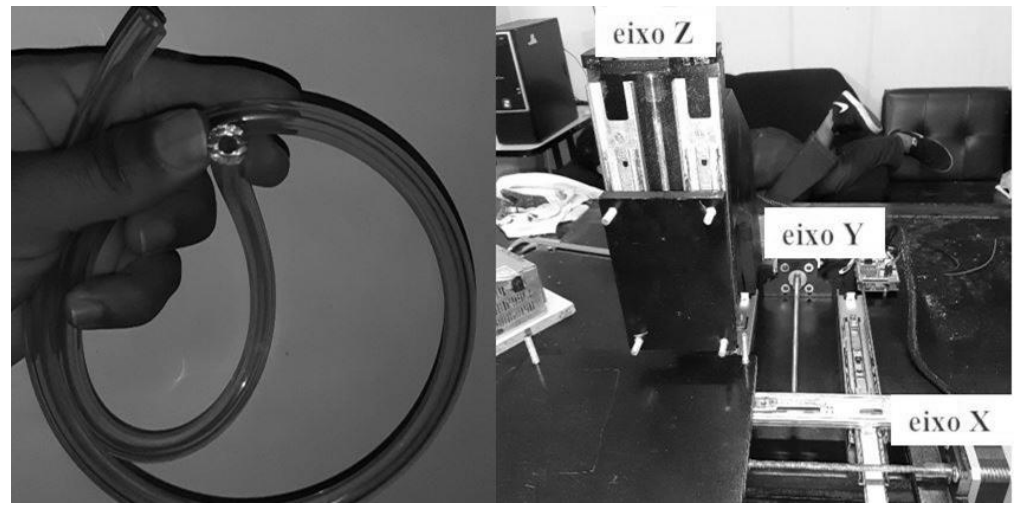

Figura 4. Mangueira para gasolina em silicone (esquerda), Eixos $X, Y$ e Z conectados com a mangueira aos motores (direita).

Fonte: Autor (2020)

Além dessas alterações, foram adicionados em cada canto da peça 6 , quatro parafusos sextavados de $1 / 4$ de polegada para a fixação da máquina-ferramenta a ser utilizada. Essa fixação se deu por meio de porcas borboletas permitindo a troca de tal ferramenta de forma simples e fácil, tendo em vista que a máquina $\mathrm{CNC}$ foi construída para ser utilizada com uma fresadora e um laser de forma não paralela. A Figura 5 mostra a forma de acoplagem dos "módulos" de máquinas-ferramentas utilizadas na máquina CNC.
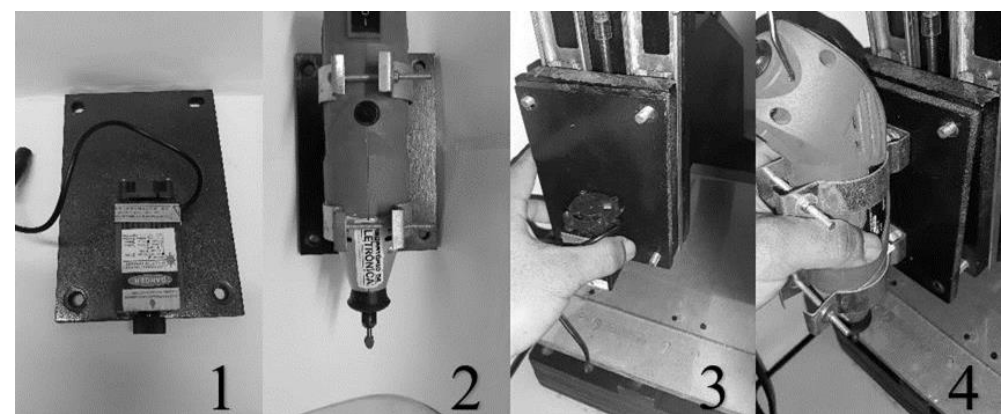

Figura 5. "Módulos" de máquinas-ferramenta (1 e 2), Suporte de encaixe dos módulos (3 e 4).

Fonte: Autor (2020) 


\subsection{Custos}

Mesmo sendo construída reutilizando materiais reciclados, a construção da máquina CNC necessitou de itens que foram obtidos por meio de compras em lojas online e físicas, tendo em vista que antes de serem adquiridas, foi necessária uma pesquisa de mercado visando a escolha do menor custo possível de materiais. Com isso, para demonstrar e validar a viabilidade de redução de custos no uso de materiais reciclados, foi necessário uma análise dos custos empregados por este projeto comparado aos projetos de Walendorff (2020), Moreira (2018) e Dantas; Junior (2016) ilustrados nas Tabela 1, 2, 3 e 4.

Tabela 1. Tabela de custo da CNC de Walendorff (2020).

\begin{tabular}{|c|c|c|}
\hline Itens & Quantidade & Subtotal \\
\hline Motores de passo NEMA $17 \mathrm{com}$ torque igual ou superior a $2.5 \mathrm{kgf} . \mathrm{cm}$. & 3 & $\mathrm{R} \$ 226,95$ \\
\hline Fonte $12 \mathrm{VDC} \times 5^{\mathrm{a}}$ & 1 & $\mathrm{R} \$ 21,00$ \\
\hline Retifica igual ou similar eccofer AR172 & 1 & $R \$ 130,00$ \\
\hline Fio $0,25 \mathrm{~mm}$ de diâmetro. Vai depender de como você montar. (metro) & 20 & 4,00 \\
\hline Arduino uno + cabo. & 1 & $\mathrm{R} \$ 39,00$ \\
\hline CNC Shield V3.0 & 1 & $R \$ 25,00$ \\
\hline Drive motor de passo A4988 & 3 & $\mathrm{R} \$ 45,66$ \\
\hline Barra roscada 1/4 (metro) & 1 & 3,00 \\
\hline Espaguete termo retrátil $9.5 \mathrm{~mm}$ de diâmetro (metro) & 0,5 & 3,00 \\
\hline Porcas para barra roscada de $1 / 4$ & 3 & 1,00 \\
\hline Módulo relé para controlar a retífica & 1 & 7,65 \\
\hline Chave Fim de Curso (Limit Switch) KW11-7-1 16A 250VAC com Haste 60mm & 6 & $\mathrm{R} \$ 20,00$ \\
\hline $\mathrm{PCl} 5 \mathrm{~cm} \times 5 \mathrm{~cm}$ & 1 & 1,19 \\
\hline \multicolumn{2}{|l|}{ VALOR TOTAL DE CUSTO } & $\mathrm{R} \$ 527,45$ \\
\hline
\end{tabular}

Tabela 2. Tabela de custo da CNC de Moreira (2018).

\begin{tabular}{|c|c|c|c|}
\hline Itens & Quantidade & \multicolumn{2}{|c|}{ Subtotal } \\
\hline Estrutura Física & 1 & $\mathrm{R} \$$ & 249,00 \\
\hline Micro Retífica AWT Red & 1 & $\mathrm{R} \$$ & 249,00 \\
\hline Raspberry Pi3 & 1 & $\mathrm{R} \$$ & 220,00 \\
\hline Fonte de Alimentação (24V 15A) & 1 & $\mathrm{R} \$$ & 80,00 \\
\hline Motores de passo (Usados) & 3 & $\mathrm{R} \$$ & 50,00 \\
\hline Driver A4988 & 3 & $\mathrm{R} \$$ & 33,00 \\
\hline Arduino UNO & 1 & $\mathrm{R} \$$ & 25,00 \\
\hline Trilhos Telescópicos & 6 & $\mathrm{R} \$$ & 34,00 \\
\hline Polia Gt2 5mm & 1 & $\mathrm{R} \$$ & 14,90 \\
\hline Polia GTt2 6.35mm & 1 & $\mathrm{R} \$$ & 14,90 \\
\hline Correia Gt2 400mm & 1 & $\mathrm{R} \$$ & 14,90 \\
\hline Abraçadeiras metálicas de $50 \mathrm{~mm}$ de diâmetro & 1 & $\mathrm{R} \$$ & 8,00 \\
\hline Barra de rosca de $1 / 4$ & 1 & $\mathrm{R} \$$ & 4,00 \\
\hline Porcas de $1 / 4$ & 6 & $\mathrm{R} \$$ & 2,00 \\
\hline Parafusos $60 \mathrm{~mm} \times 4 \mathrm{~mm}$ & 2 & $\mathrm{R} \$$ & 0,40 \\
\hline Parafusos $80 \mathrm{~mm} \times 4 \mathrm{~mm}$ & 2 & $\mathrm{R} \$$ & 0,40 \\
\hline Parafusos $40 \mathrm{~mm} \times 4 \mathrm{~mm}$ & 4 & $\mathrm{R} \$$ & 0,60 \\
\hline Parafusos $14 \mathrm{~mm} \times 4 \mathrm{~mm}$ & 25 & $\mathrm{R} \$$ & 2,50 \\
\hline Acoplador flexível de motor & 2 & $\mathrm{R} \$$ & 29,80 \\
\hline Fresa $60^{\circ} 0,1 \mathrm{~mm}$ & 1 & $\mathrm{R} \$$ & 14,90 \\
\hline Placa de fenolite $20 \mathrm{~cm} \times 20 \mathrm{~cm}$ & 1 & $\mathrm{R} \$$ & 12,00 \\
\hline VALOR TOTAL DE CUSTO & & $\mathbf{R} \$$ & $1.059,30$ \\
\hline
\end{tabular}

Tabela 3. Tabela de custo da CNC de Dantas; Junior (2016).

\begin{tabular}{|c|c|c|c|}
\hline Itens & Quantidade & \multicolumn{2}{|c|}{ Subtotal } \\
\hline CNC Shield & 1 & $\mathrm{R} \$$ & 5,23 \\
\hline Driver A4988 & 3 & $\mathrm{R} \$$ & 13,26 \\
\hline Motor de Passo NEMA 17 & 3 & $\mathrm{R} \$$ & 115,14 \\
\hline Arduino UNO & 1 & $\mathrm{R} \$$ & 12,81 \\
\hline Fonte Chaveada Industrial 60W 12V 5A & 1 & $\mathrm{R} \$$ & 48,30 \\
\hline Barra roscada de 1/4 (metro) & 2 & $\mathrm{R} \$$ & 12,60 \\
\hline Porcas de $1 / 4$ & 3 & $\mathrm{R} \$$ & 0,30 \\
\hline Trilho Telescópico Light (par) & 3 & $\mathrm{R} \$$ & 20,10 \\
\hline Micro Retífica & 1 & $\mathrm{R} \$$ & 229,00 \\
\hline Acoplador Sanfonado & 3 & $\mathrm{R} \$$ & 10,92 \\
\hline
\end{tabular}


VALOR TOTAL DE CUSTO

$\mathbf{R} \$ \quad 467,66$

Tabela 4. Tabela de custo do projeto REM-CNC Router, descrito neste trabalho.

\begin{tabular}{|c|c|c|c|}
\hline Itens & Quantidade & \multicolumn{2}{|c|}{ Subtotal } \\
\hline Estrutura Física & 1 & $\mathrm{R} \$$ & 165,00 \\
\hline Arduino UNO REV3 & 1 & $\mathrm{R} \$$ & 35,70 \\
\hline CNC Shield & 1 & $\mathrm{R} \$$ & 17,90 \\
\hline Graxa Branca & 1 & $\mathrm{R} \$$ & 5,00 \\
\hline Trilho telescópico $25 \mathrm{~cm}$ (par) & 2 & $\mathrm{R} \$$ & 60,00 \\
\hline Adesivo Instantâneo 793 TEKBOND & 1 & $\mathrm{R} \$$ & 10,00 \\
\hline Driver A4988 & 3 & $\mathrm{R} \$$ & 26,10 \\
\hline Motor de Passo NEMA 17 & 3 & \multicolumn{2}{|c|}{ Reciclado } \\
\hline Fonte Bematech Mp20 Fr20 Ft2997 - 24V & 1 & \multicolumn{2}{|c|}{ Reciclado } \\
\hline Módulo Relé & 1 & \multicolumn{2}{|c|}{ Reciclado } \\
\hline Barra Roscada 1/4 (metro) & 1 & $\mathrm{R} \$$ & 2,30 \\
\hline Parafuso madeira $3,5 \times 30 \mathrm{~mm}$ & 8 & $\mathrm{R} \$$ & 0,80 \\
\hline Parafuso madeira $3 \times 30 \mathrm{~mm}$ & 4 & $\mathrm{R} \$$ & 0,40 \\
\hline Parafuso madeira $3,5 \times 25 \mathrm{~mm}$ & 2 & $\mathrm{R} \$$ & 0,20 \\
\hline Parafuso madeira $6 \times 65 \mathrm{~mm}$ & 12 & $\mathrm{R} \$$ & 6,00 \\
\hline Parafuso madeira $4 \times 20 \mathrm{~mm}$ & 4 & $\mathrm{R} \$$ & 0,20 \\
\hline Parafuso madeira $3,5 \times 20 \mathrm{~mm}$ & 14 & $\mathrm{R} \$$ & 0,70 \\
\hline Parafuso madeira $3 \times 16 \mathrm{~mm}$ & 10 & $\mathrm{R} \$$ & 0,50 \\
\hline Parafuso madeira $4,5 \times 25 \mathrm{~mm}$ & 4 & $\mathrm{R} \$$ & 0,40 \\
\hline Parafuso Sextavado Zincado UNC 1/4"x1.1/4" & 4 & $\mathrm{R} \$$ & 1,20 \\
\hline Parafuso Sextavado $6 \times 50 \mathrm{~mm}$ & 2 & $\mathrm{R} \$$ & 0,80 \\
\hline Porca $6 \mathrm{~mm}$ & 2 & $\mathrm{R} \$$ & 0,10 \\
\hline Porca $1 / 4$ & 6 & $\mathrm{R} \$$ & 0,60 \\
\hline Porca Borboleta 1/4 & 4 & $\mathrm{R} \$$ & 2,80 \\
\hline Mangueira para gasolina (metro) & 1 & $\mathrm{R} \$$ & 5,00 \\
\hline Abraçadeira Inca Tipo D2 & 2 & $\mathrm{R} \$$ & 10,00 \\
\hline VALOR TOTAL DE CUSTO & & $\mathbf{R} \mathbf{S}$ & 351,70 \\
\hline
\end{tabular}

Com base nos valores mostrados nas Tabelas de custo, podemos notar as diferenças entre os recursos gastos para a construção de um projeto baseado no projeto de Walendorff (2020), tendo em vista que esse valor pode variar conforme o tamanho da área de trabalho, a variação dos tipos de máquinas-ferramentas que serão utilizadas e a forma de uso da máquina $\mathrm{CNC}$.

\section{Resultados e Discussões}

Após todo o processo de montagem e as alterações para melhoria da máquina $\mathrm{CNC}$, pôde ser obtido uma máquina $\mathrm{CNC}$ de baixo custo com parte dos materiais usados provenientes de resíduo eletrônico. Isso permitiu a redução dos custos, em relação ao projeto original que já era de baixo custo, mostrando que há um grande potencial na reutilização de resíduos eletrônicos na construção de máquinas $\mathrm{CNC}$ com custo reduzido.

Assim que foi construída, a máquina $\mathrm{CNC}$ foi utilizada para fazer placas de circuito impresso e gravações a laser em superfícies de alguns materiais, ilustrados na Figura 6. A REM-CNC Router fez parte de uma exposição no MT Ciências no Sesc em CuiabáMT, demostrando para a comunidade local a importância de reutilização de materiais reciclados para a construção de tais projetos e está sendo utilizada na produção de máscaras protetoras do tipo Face Shield para auxiliar no combate ao Covid-19. 


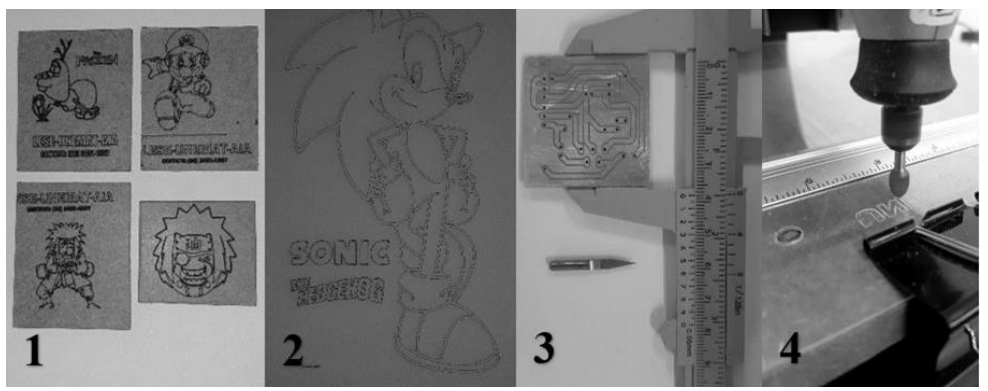

Figura 6. Resultado do uso da CNC ( 1 e 2 feita a laser) e (3 e 4 feita com fresa).

Fonte: Autor (2020).

Para analisar a precisão da máquina $\mathrm{CNC}$, foram efetuados alguns testes utilizando a ferramenta laser e uma placa de MDF em espessura de $3 \mathrm{~mm}$. Sabendo que o raio laser acoplado na máquina possui $0,05 \mathrm{~mm}$ de diâmetro, foram traçadas de 5 à 13 linhas paralelas por milímetro (mm) com $14 \mathrm{~mm}$ de comprimento (Figura 7).

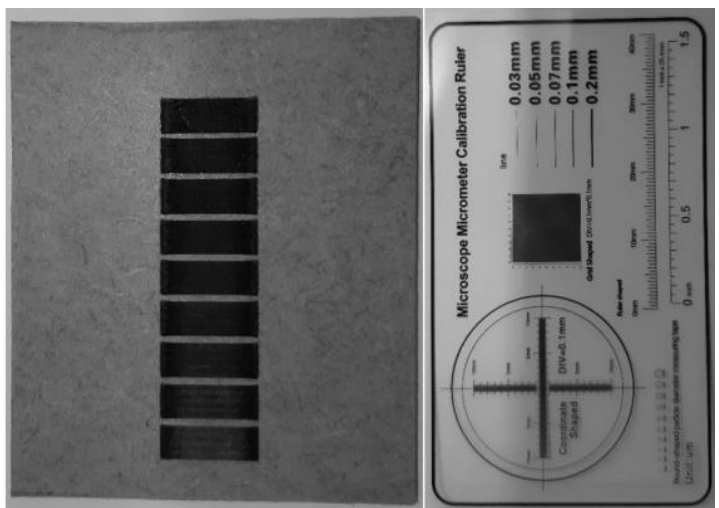

Figura 7. Placa de MDF com retângulos de $5 \mathrm{~mm} \times 14 \mathrm{~mm}$ gravados a laser (esquerda) e régua de escala da câmera microscópica (direita).

Fonte: Autor (2020).

Utilizando uma câmera microscópica, foi possível analisar a resolução e a precisão alcançada pela máquina $\mathrm{CNC}$, sendo que a maior precisão que pôde ser alcançada foi de $50 \mu \mathrm{m}$ com uma resolução de $100 \mu \mathrm{m}$, que pode ser analisada na Figura $8 \mathrm{com}$ a escala baseada na régua $(500 \mu \mathrm{m})$ da Figura 7 .

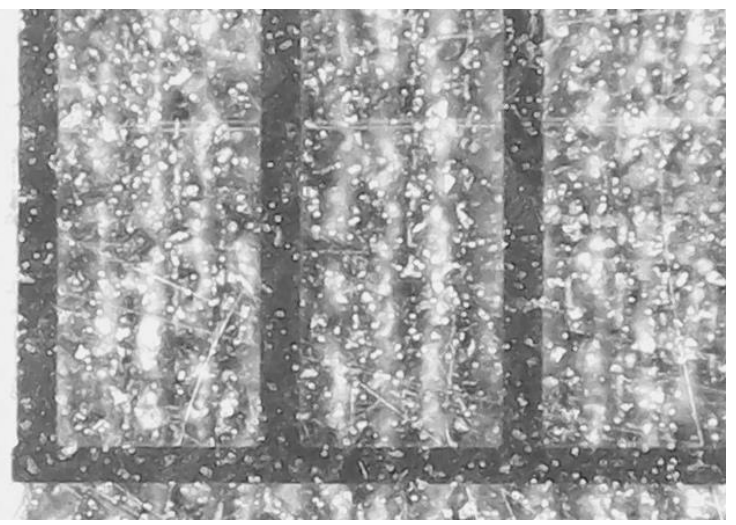

Figura 8. Imagem aumentada da Placa de MDF com retângulos de $5 \mathrm{~mm} x$ $14 \mathrm{~mm}$ gravados a laser.

Fonte: Autor (2020). 


\section{Conclusões}

Com base nos custos aplicados neste projeto e os resultados obtidos, podemos afirmar que a REM-CNC Router é uma máquina CNC de baixo custo, quando comparada com outras máquinas $\mathrm{CNC}$ que desempenham as mesmas funcionalidades. A REM-CNC Router possui como principal vantagem a capacidade de operar dois tipos de ferramentas (não simultaneamente) um Laser, capaz de fazer gravações na superfície de materiais; e uma Micro retifica, permitindo além da gravação na superfície de materiais, a usinagem de peças $2 \mathrm{D}$ e até mesmo 3D. Outra vantagem é a área de trabalho maior quando comparada com as similares encontradas na literatura. A REM-CNC Router é uma máquina $\mathrm{CNC}$ multifuncional, construída com materiais reciclados, de um custo reduzido e precisão micrométrica, comprovando que o uso desses materiais reduz os custos de produção de máquinas $\mathrm{CNC}$.

\section{Referências}

ANIS, Adie; SANTOSA, Irfan. Instrumen Kendali Mesin CNC Portable Berbasis Microcontroller Arduino dan Modul CNC Shield. 2019. 1st Mechanical Engineering Conference [...]. [S. l.: s. n.], 2019.

ARDUINO. What is Arduino? 2020. Disponível em: https://www.arduino.cc/en/Guide/Introduction. Acesso em: 17 ago. 2020.

ARDUINO UNO REV3 | LOJA OFICIAL DO ARDUINO. 2020. Disponível em: https://store.arduino.cc/usa/arduino-uno-rev3. Acesso em: 10 ago. 2020.

CARDOSO JÚNIOR, Edinaldo Serra; OBANA, Fernando Yoiti; SOUZA, Feliphe Carvalho Rodrigues de; NASCIMENTO, Leonardo Xavier do; SPEROTTO, Lucas Kriesel; MARINHO, Max Robert. Floppy Music V2 - um sistema robótico de DEC capaz de reproduzir músicas integrado a um módulo Mixer de Áudio com Préamplificação. 2019. Anais do Congresso de Ensino, Pesquisa e Extensão. Disponível em: https://eventos.ifmt.edu.br/publicacao/569/. Acesso em: 8 ago. 2020.

DANTAS, Marcus Paulo Soares; JUNIOR, Orivaldo Vieira De Santana. Fresadora CNC de baixo custo-SUSY. Trabalho apresentado na Mostra Nacional de Robótica (MNR), 2016.

FACHIM, Alan. Projeto de fresadora CNC com plataforma livre Arduino. 2013.

GOBI, Nathan. Desenvolvimento de protótipo de máquina CNC de baixo custo para processos de corte e gravação em micro e pequenas empresas. 2019. B.S. thesis 2019.

JAYACHANDRAIAH, B.; KRISHNA, O. Vamsi; KHAN, P. Abdullah; REDDY, R. Ananda. Fabrication of low cost 3 -Axis $\mathrm{CNC}$ router. International Journal of Engineering Science Invention, v. 3, n. 6, p. 1-10, 2014. 
LASERGRBL. LaserGRBL - Gravação a laser gratuita. 2020. Disponível em: http://lasergrbl.com/. Acesso em: 19 ago. 2020.

LEITE, Josicleudo R.; LEITE, Josileudo R.; SANTOS\$^1\$, Josiel L.; MACÊDO, Christiane B.; BAIER, Pablo AS; DO NORTE, Limoeiro. DESENVOLVIMENTO DE UM CNC DE PEQUENO PORTE. Reunião Regional da SBPC no Cariri URCA - Cariri/CE, 2017. .

MOREIRA, Divino Luiz Barbosa. Construção de uma fresa de controle númerico computadorizado para placas de circuito impresso. 2018. .

OBANA, Fernando Yoiti; SPEROTTO, Lucas Kriesel; MARINHO, Max Robert; DOS SANTOS, Raiane Talissa. REUTILIZAÇÃO E RECICLAGEM DE EQUIPAMENTOS DE INFORMÁTICA EM UMA CIDADE DE PEQUENO PORTE. Revista Compartilhar-Reitoria, v. 3, n. 1, p. 63-69, 2018. .

PALMIERE, Sérgio Eduardo. CLP versus Microcontrolador - Embarcados. $11 \mathrm{fev}$. 2016. Embarcados - Sua fonte de informações sobre Sistemas Embarcados. Disponível em: https://www.embarcados.com.br/clp-versus-microcontrolador/. Acesso em: 10 ago. 2020.

POLASTRINI, HF. Desenvolvimento de uma Máquina Cnc de Baixo Custo com Software e Hardware Abertos. Relatório (Graduando em Engenharia Elétrica), Instituto Federal de Minas Gerais, 2016. .

REZENDE, Henrique B; BASTOS, Maria Eduarda S; LANES, Matusalém M; PIRES, Anderson G. FERRAMENTA COMPUTACIONAL PARA CONTROLE DE MÁQUINAS CNC. , p. 8, 2019. .

VERMA, Kushal; GIANCHANDANI, Ishita; SAROHA, Sumit. Efficient Use of Electronic Waste for CNC Machine. International Journal of Computer Applications, v. 975, p. 8887, 2018. .

WALENDORFF, Marlon Nadi. Construa sua própria CNC 2.1. 2020. Disponível em: https://www.marlonnardi.com/p/construa-sua-propria-cnc-20-2.html. Acesso em: 7 ago. 2020. 